\title{
Ectopia "cordis": relato de 2 casos operados. Discussão do manuseio cirúrgico e revisão da literatura
}

Mozart ESCOBAR * Ricardo de Carvalho LIMA * Nadja Arraes FRANÇA * , Haydee BELÉM *, Eugênia SCHMIDT *, Maria P. SOUZA *, Roberto ALECRIM *, Renato DELLA SANTA *, Nereide MELLO *, Luiz G. GRANJA *, Joel PONTES *

ESCOBAR, M.; LIMA, R. C.; FRANÇA, N. A.; BELÉM, H.; SCHMIDT, E.; SOUZA, M.P.; ALECRIM, R.; DELLA SANTA, R.; MELLO, N.; GRANJA, L. G.; PONTES, J. - Ectopia "cordis": relato de 2 casos operados. Discussão do manuseio cirúrgico e revisão da literatura. Rev. Bras. Cir. Cardiovasc., 8 (3): 241-246, 1993.

RESUMO: A ectopia "cordis" verdadeira é uma doença congênita, rara e caracterizada pela presença do coração fora do tórax, havendo, como principal característica, a ausência das camadas que o recobrem. $\mathrm{Na}$ grande maioria dos pacientes portadores da ectopia "cordis" verdadeira existe associação de defeitos intracardiacos complexos. No presente trabalho, os autores relatam sua experiência com 2 casos operados, na Unidade de Tratamento Cardiotorácico - UNITÓRAX - do Real Hospital Português do Recife, bem como discutem o manuseio cirúrgico e fazem uma revisão da literatura.

DESCRITORES: ectopia "cordis", cirurgia.

\section{INTRODUÇÃO}

A ectopia "cordis" verdadeira é uma entidade rara onde o coração encontra-se totalmente fora do tórax, tendo como principal característica a ausência de todas as camadas que o recobrem (pericárdio, esterno, músculos e pele). Normalmente, esta doença congênita está associada a defeitos intracardíacos complexos e a uma alta mortalidade.

O primeiro caso de ectopia "cordis" relatado na literatura foi descrito por Neil Stensen, em 1671 (citado por MILLHOUSE \& JOOS ${ }^{1}$ ). Este paciente apresentava, ainda, uma tetralogia de Fallot.

Os autores apresentam 2 casos de correção cirúrgica da ectopia "cordis" verdadeira com o diagnóstico no período intra-uterino, operados na Unidade de Tratamento Cardiotorácico - UNITÓRAX do Real Hospital Português do Recife, e fazem consi- derações sobre o manuseio cirúrgico e revisão da literatura.

Caso de n 1:

E. V. R., 22 anos, sexo feminino, casada, mãe de uma menina com 2 anos de idade, inteiramente normal. $\mathrm{Na}$ evolução da segunda gestação (sem fatores teratogênicos), a paciente, quando se encontrava no decurso da décima semana, fez controle ultra-sonográfico, revelador de uma pequena área de descolamento do saco gestacional, com hematoma. Na semana seguinte, um novo controle evidenciou acentuada diminuição da área do hematoma.

$\mathrm{Na}$ trigésima quinta semana, fez novo controle com ultrasom, que demonstrou maturidade placentária grau I com $67 \%$ de maturidade fetal, havendo

Trabalho realizado no UNITÓRAX do Real Hospital Português do Recife, Recife, PE.

Apresentado ao $20^{2}$ Congresso Nacional de Cirurgia Cardiaca. Maceió, AL, de abril, 1993.

- Do UNITÓRAX.

Endereço para separatas: Rua Neil Amstrong 43/401, 52060-170, Recife, PE. 
ESCOBAR, M.; LIMA, R. C.; FRANÇA, N. A.; BELÉM, H.; SCHMIDT, E.; SOUZA, M.P.; ALECRIM, R.; DELLA SANTA, R.; MELLO, N.; GRANJA, L. G.; PONTES, J. - Ectopia "cordis": relato de 2 casos operados. Discussão do manuseio cirúrgico e revisão da literatura. Rev. Bras. Cir. Cardiovasc., 8 (3): 241-246, 1993.

apresentação cefálica com dorso anterior à direita, presença de movimentos fetais e movimentos cardíacos positivos. Durante este exame, foi detectada massa pulsátil anterior ao tórax, sendo diagnosticada como ectopia "cordis".

Inconformados com o diagnóstico, os familiares da paciente procuraram outra clínica especializada, realizando outro exame, o qual concluiu haver massa pulsátil condizente com ectopia "cordis" em conexão com a parede anterior do tórax, na linha média através de estruturas vasculares de grosso calibre (Figura 1).

Em 7.7.1986, a paciente entrou em trabalho de parto, sendo submetida, em caráter de urgência, a uma cesariana, quando nasceu um feto a termo, do sexo feminino, apresentando como única deformidade a ectopia "cordis" verdadeira e uma onfalocele. A recém-nascida foi preparada para a cirurgia.

A operação consistiu na dissecção do pedículo vascular identificando-se cada vaso. Encontramos um vaso arterial, que, saindo do coração, adentrava o tórax, dando dois ramos em direção à cabeça. Este vaso continuava para baixo, após fazer ligeira curva para o interior do corpo. Um outro vaso, único, era originário do interior do corpo, caminhando em direção ao coração e, aí, penetrando junto ao vaso anteriormente descrito.

Com o pedículo vascular liberado e suas estruturas identificadas, ambas as pleuras foram abertas para dar maior liberdade ao coração. Esta manobra não permitiu um bom posicionamento do coração, tendo sido necessária a ressecção da metade inferior esquerda do esterno.

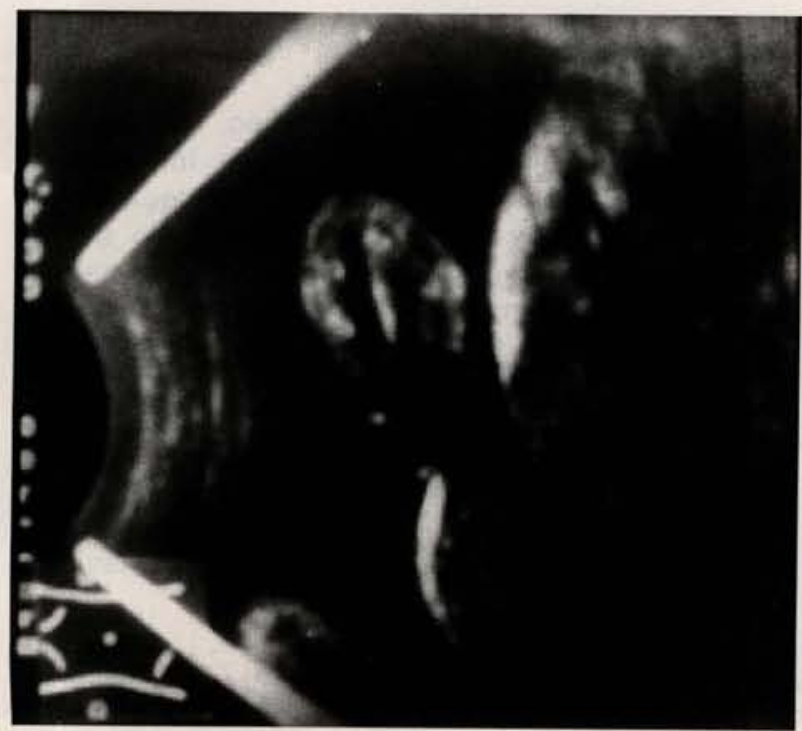

Fig. 1 - Uitra-som: presença da ectopia "cordis". Pode-se suspeitar da presença de um único átrio.

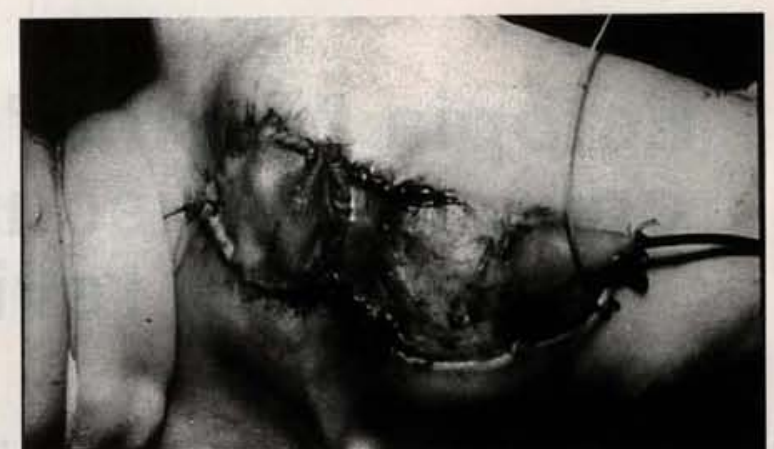

Fig. 2 - Aspecto final da operação. O orificio foi fechado com malha de Tefion.

O fechamento do tórax, depois da drenagem de ambas as pleuras, foi conseguido da dissecção do folheto anterior da onfalocele, rebatido superiormente e suturado à pele. $\mathrm{Na}$ região correspodente ao orifício de saída dos vasos e do coração, conseguimos, com sucesso, o seu fechamento, usando um enxerto de malhas de Teflon suturado à pele (Figura 2).

Terminada a operação, a criança foi levada à sala de tratamento intensivo sob respiração controlada, apresentando excelentes parêmetros hemodinâmicos. Na décima hora de pós-operatório, iniciouse quadro de intensa acidose metabólica de difícil controle, culminando com o óbito na décima quinta hora do pós-operatório.

O exame anatomopatológico do coração mostrou grave cardiopatia congênita, que consistiu em uma única valva atrioventricular, ventrículo direito aumentado, ventrículo esquerdo hipoplásico (talvez rudimentar), presença de comunicação interventricular e hipoplasia da aorta (Figura 3).

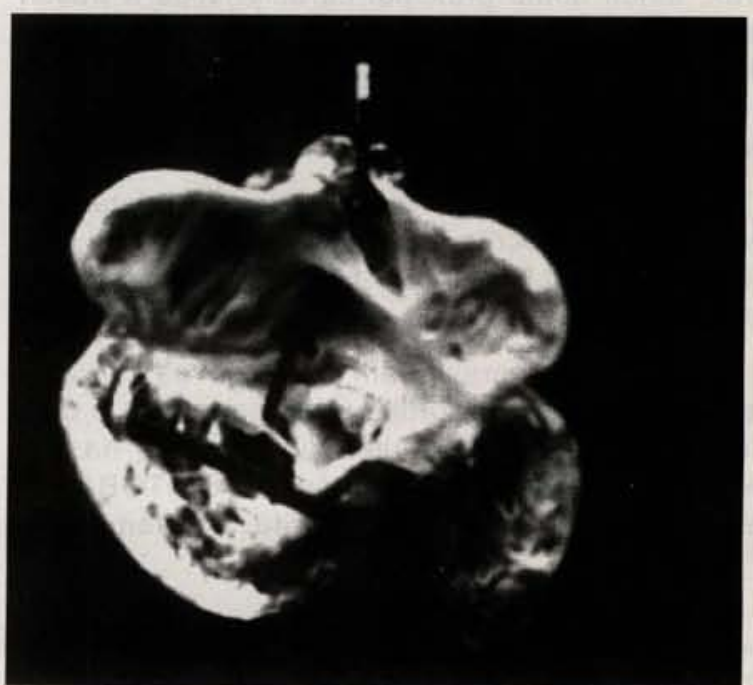

Fig. 3 - Aspecto do coraçăo: presença de um unico átrio; presença da CIV e ventrículo esquerdo hipoplástico. 
ESCOBAR, M.; LIMA, R. C.; FRANÇA, N. A.; BELÉM, H.; SCHMIDT, E.; SOUZA, M. P.; ALECRIM, R.; DELLA SANTA, R.; MELLO, N.; GRANJA, L. G.; PONTES, J. - Ectopia "cordis": relato de 2 casos operados. Discussão do manuseio cirúrgico e revisão da literatura. Rev. Bras. Cir. Cardiovasc., 8 (3): 241-246, 1993.

\section{Caso de n 2:}

Gestante jovem, da cidade de Garanhuns, ao final do terceiro trimestre realizou, em sua cidade, controle ultra-sonográfico para avaliação, rotineira, da gestação. O exame mostrou feto com o coração fora do tórax (Figura 4). A paciente foi orientada a programar um parto cesáreo em hospital com capacidade para realizar a correção cirúrgica da anomalia imediatamente após o nascimento da criança.

Decorridos 17 dias após a realização do referido exame, a paciente não deu ouvidos às recomendações médicas quanto ao parto, resolvendo ficar em sua cidade, que dista de nossa cidade aproximadamente $200 \mathrm{Km}$. Entrou em trabalho de parto, sendo submetida a uma cesareana, da qual nasceu uma criança de sexo masculino, passando 2.500 gramas, apresentando uma ectopia "cordis" verdadeira.

A presença da malformação motivou, de imediato, a transferência do recém-nascido para o nosso Serviço. Vale ressaltar que a dita transferência se deu nas piores condições possíveis, havendo grave contaminação da área exposta.

$\mathrm{Na}$ avaliação clínica inicial, tratava-se de uma criança morfologicamente normal, tendo, como única anormalidade, a presença do coração fora do tórax. Encontrava-se cianótica, taquipnéica e com freqüência cardíaca de $100 \mathrm{bpm}$. O coração situava-se fora do tórax em aproximadamente $2 / 3$, não havendo formação do esterno.

O aspecto macroscópico do coração foi considerado normal, como também a relação ventrículoarterial.

Realizamos um ecocardiograma bidimensional (Figura 5) em condições estéreis, que não evidenciou anomalias estruturais das câmaras cardiacas, vasos e septos. Também foi realizado exame radi-

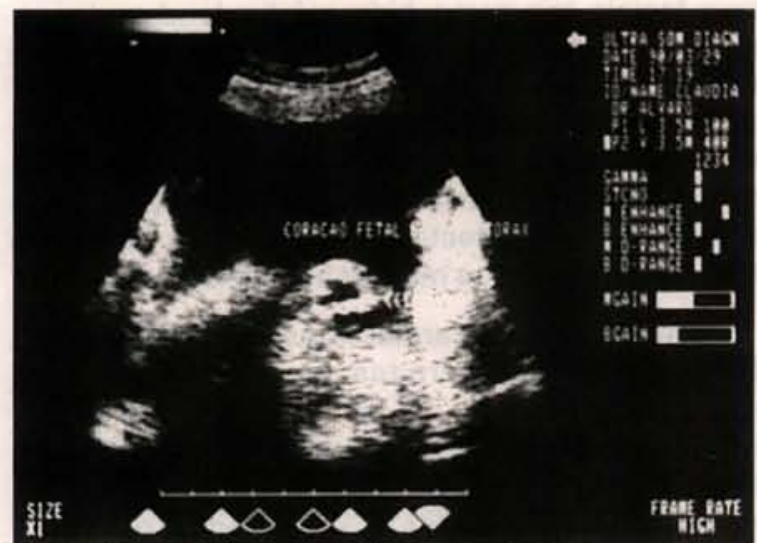

Fig 4 - Caso 2: Ultra-sonografia, mostrando presença do coraçăo fora do tórax.

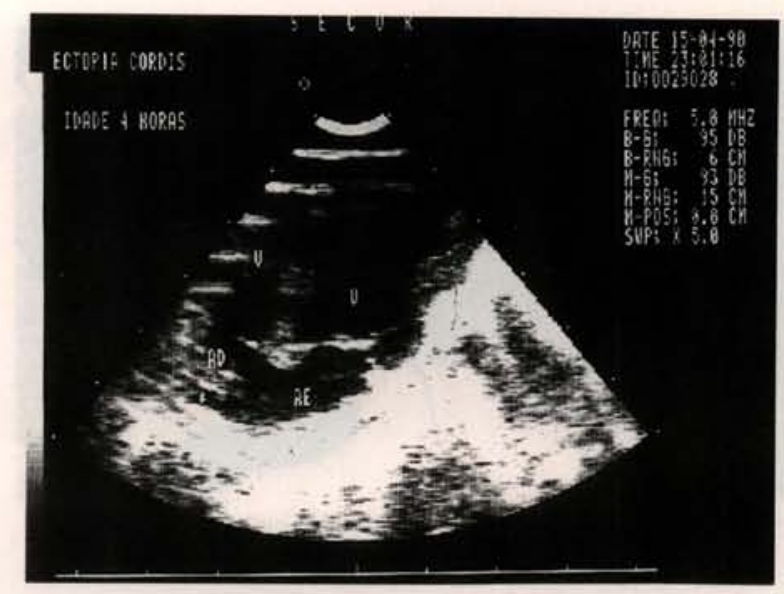

Fig. 5 - Ecocardiograma realizado no caso 2, não evidenciando anomalias intracardiacas.

ológico do tórax em projeção ântero-posterior, que revelou ausência dos arcos costais anteriores e do esterno. A criança ficou em ambiente estéril em uma incubadoura, enquanto aguardava a operação.

Após a anestesia geral, foram dissecadas a artéria radial, para monitorização da pressão arterial média, e a veia cefálica, para introdução de drogas e reposição da volemia.

A cirurgia consistiu em uma incisão mediana do tórax abrangendo o local por onde o coração se exteriorizava, para cima até a fúrcula esternal e para baixo até a altura do diafragma. O coração teve seus vasos da base totalmente dissecados e liberados o bastante para que não angulasse quando do seu reposicionamento dentro do tórax. A pleura mediastínica esquerda foi amplamente aberta, para permitir um espaço maior ao coração, que ficou repousado sobre o diafragma.

Após este tempo cirúrgico, a equipe de cirurgia plástica procedeu ao fechamento do tórax. Este foi conseguido por uma ampla dissecção dos músculos peitorais, bilateralmente, até seus pedículos vasculares. Com a musculatura peitoral livre, foram rodados, primeiramente o peitoral esquerdo, em direção ao lado contrário e aí fixado. Posteriormente, o peitoral direito passando por cima do anterior e fixado através de uma sutura simples do lado contrário. Ao término deste rocedimento, a linha mediana do tórax ficou totalmente fechada pelos dois grandes músculos, dando melhor estabilidade à parede torácica. A pele foi suturada recobrindo a camada muscular. Obteve-se, assim, um excelente resultado plástico, sem que houvesse compressão do coração, angulação de seus vasos ou, mesmo, aumento da pressão intratorácica dificultando a expansão pulmonar (Figura 6).

Estes parâmetros foram analisados observando-se a estabilidade hemodinâmica, como também 
ESCOBAR, M.; LIMA, R. C.; FRANÇA, N. A.; BELÉM, H.; SCHMIDT, E.; SOUZA, M. P.; ALECRIM, R.; DELLA SANTA, R.; MELLO, N.: GRANJA, L. G.; PONTES, J. - Ectopia "cordis": relato de 2 casos operados. Discussão do manuseio cirúrgico e revisão da literatura. Rev. Bras. Cir. Cardiovasc., 8 (3): 241-246, 1993.

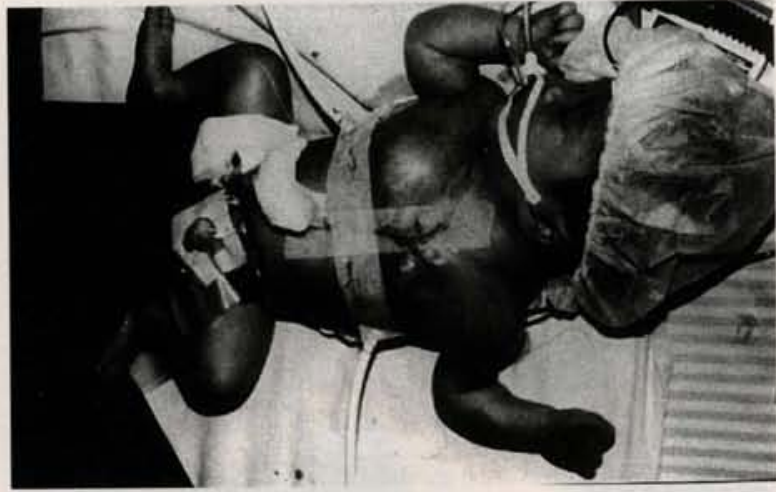

Fig. 6 - Caso 2: aspecto final da correção cirúrgica.

a observação da pressão intrapulmonar mostrada pelo respirador.

Ao término da cirurgia, a criança foi conduzida à UTI, onde ficou sob observação. Apresentou excelente evolução nas primeiras 24 horas, mas, a partir daí, iniciou quadro compatível com processo infeccioso generalizado, vindo a falecer na quinquagésima segunda hora de pós-operatório, em septicemia.

\section{COMENTÁRIOS}

A grande maioria dos defeitos da parede torácica traz pouca repercussão funcional, embora provoque grandes alterações estéticas. Existe porém um grupo de defeitos que podem provocar graves problemas hemodinâmicos e respiratórios. Entre eles, encontramos a falta de fusão do esterno, provocando instabilidade da parede torácica, o que levará, conseqüentemente, à insuficiência respiratória.

Em decorrência desta situação, podemos encontrar o coração projetado para fora do tórax. recoberto ou não de sua proteção natural 16,18 .

A presença deste defeito parece estar associada a uma compressão mecânica secundária à rotura do córion e ou saco vitelino, por volta da terceira semana de gestação. Esta compressão interfere na descida normal do coração, comprime o tórax e não permite a fusão do esterno em formação. Parece não haver relação do defeito torácico com prováveis presenças de trissomias, como foi demonstrado por KHOURY et alii ${ }^{12}$.

Durante a sexta semana, o embrião apresenta duas formações esternais em posição ventro-lateral, originadas do mesênquima e separadas entre si na linha média. Durante as três semanas seguintes, estas duas barras cartilaginosas se unem aos arcos costais, iniciando-se, assim, a fusão progressiva de ambas em sentido cefálocaudal, originando o esterno e, conseqüentemente, dando-se o fechamento da linha média do corpo $1,3,4,19$.

Esta dupla origem do esterno fechando-se na linha média explica o porquê da localização da ectopia "cordis" em: cervical cervicotorácica, torácica, toracoabdominal e abdominal, sendo a forma torácica a mais freqüente, representando $37 \%$ dos casos relatados na literatura 5,14 .

Recentemente, o diagnóstico da ectopia "cordis" ou, mesmo, de outros defeitos na formação do feto pode ser realizado mais precocemente ainda, já que novas técnicas ultra-sonográficas e o mais moderno aparelhamento permitem detectar anomalias com maior precisão intra-útero, facilitando a programação assistêncial para crianças com doenças tão graves $6,9,13,21$. Em nossos 2 enfermos, a presença da ectopia "cordis" foi diagnósticada através do ultrasom. No primeiro caso, a nitidez do exame permitiu suspeitar da presença de grave cardiopatia onde se viu apenas uma valva atrioventricular e a possibilidade de um ventrículo hipoplásico.

Nos 2 pacientes não foi possível estabelecer um padrão eletrocardiográfico em virtude da urgência e do temor de contaminação do coração exposto. Este procedimento já está relatado na literatura médica, mostrando que o sistema de condução do coração se encontra normoposicionado, tendo as várias derivações demonstrado anormalidades em função, apenas, do malposicionamento do coração, representado por baixa voltagem 2,17 .

O estudo radiológico do tórax mostra ausência completa do esterno ou apenas fina barra cartilaginosa bilateral.

A ectopia "cordis" encontra-se associada a 2 tipos de anomalias: as não cardíacas e as cardíacas. As anomalias não cardíacas são freqüentes e encontram-se em aproximadamente $70 \%$ dos casos relatados. São, em sua maioria, anormalidades crânio-faciais, tais como: hidrocefalia, fenda palatina, fenda labial. Quanto ao abdome, encontramos a presença da onfalocele em $60 \%$ dos casos, aproximadamente ${ }^{11}$. Em nossos dois pacientes o primeiro não tinha anormalidades crânio-faciais, mas, em compensação, havia uma grande onfalocele. No segundo, não foi detectado nenhum tipo de defeito morfológico do corpo, a não ser a presença da ectopia.

As malformações cardíacas associadas à ectopia "cordis" foram encontradas em 95\% dos casos documentados, sendo, via de regra, hemodinamicamente importantes necessitando de correção. As formas comuns de doenças cardiacas associadas são: tetralogia de Fallot e os defeitos do septo interventricular $7,10,20$. Nossos pacientes apresentaram: o primeiro, grave defeito intracardíaco repre- 
ESCOBAR, M.; LIMA, R. C.; FRANÇA, N. A.; BELÉM, H.; SCHMIDT, E.; SOUZA, M. P.; ALECRIM, R.; DELLA SANTA, R.; MELLO, N.; GRANJA, L. G.; PONTES, J. - Ectopia "cordis": relato de 2 casos operados. Discussão do manuseio cirúrgico e revisão da literatura. Rev. Bras. Cir. Cardiovasc., 8 (3): 241-246, 1993.

sentado por uma única valva atrioventricular, ventrículo direito aumentado, com ventrículo esquerdo hipoplásico (talvez rudimentar), presença de comunicação interventricular e hipoplasia da aorta (Figura 6). O segundo tinha o coração normal. Devemos ressaltar que este paciente é o sétimo enfermo da literatura mundial com coração normal.

Embora estejam descritas várias formas de tratamento da ectopia "cordis", somos de opinião que cada caso seja estudado e tratado de acordo com a forma apresentada da doença.

Um bom planejamento cirúrgico e um preciso diagnóstico da cardiopatia associada devem ser cuidadosamente realizados, já que o diagnóstico do defeito vem sendo feito intra-útero, o que vem aumentando a freqüência da doença. É necessário que o parto seja realizado através de uma operação cesariana. Dentro do possível, o parto deve ser realizado em um mesmo bloco cirúrgico e em salas contíguas, para evitar contaminação do coração exposto. $\mathrm{Na}$ equipe de atendimento cirúrgico é necessária a presença do cirurgião plástico, para as rotações dos músculos e o reparo da pele, recobrindo a fenda cirúrgica 8,22 . Esta técnica foi empregada no nosso segundo paciente e demonstrou ser de excelente qualidade já que não provocou compressão torácica com interferência na expansão pulmo- nar, além de ter apresentado excelente aspecto estético.

De acordo com a nossa experiência e a revisão da literatura, podemos dizer:

- Que os casos de ectopia "cordis" verdadeira não ultrapassam $250 \mathrm{em}$ todo o mundo. No nosso meio, além dos 2 casos aqui apresentados, existem 3 comunicados verbalmente a nós: Dr. Mauro Arruda (Recife); Dr. Dib Tajra (Terezina) e Dr. Nilzo Ribeiro (Salvador).

- Condições, rigorosas, de assepsia para o parto e transporte do recém-nascido.

- Diagnóstico preciso do defeito intracardíaco. A oportunidade de correção deste defeito, concomitante ou não com a cirurgia reconstrutora da parede torácica, deve ser avaliada pela equipe cirúrgica.

- Equipe de cirurgia torácica deve contar com a colaboração de cirurgiões pediátricos e cirurgiōes plásticos.

- Excelente suporte pós-operatório para o manuseio do recém-nascido grave e de alto risco.

- Não usar próteses artificiais para recobrir o defeito torácico.

\section{RBCCV 44205-220}

ESCOBAR, M.; LIMA, R. C.; FRANÇA, N. A.; BELÉM, H.; SCHMIDT, E.; SOUZA, M. P.; ALECRIM, R.; DELLA SANTA, R.; MELLO, N.; GRANJA, L. G.; PONTES, J. - Truly Cordis Ectopy experience with cases and literature revision. Rev. Bras. Cir. Cardiovasc., 8 (3): 241-246, 1993.

ABSTRACT: Truly Cordis Ectopy is a rare congenital heart disease, which is caracterized by the presence of the heart out of the thoracic box, having as the main feature the absence of tissues which cover it. In the great majorly of patients with Truly Cordis Ectopy there is an association of complex intra-cardiac defects. In the present work, the authors show their experience with 2 cases who had undergone to surgery, at the Unidade de Tratamento Cardiotorácico - UNITÓRAX - in Real Hospital Português do Recife, and discuss the surgical management as well was a literature revision.

DESCRIPTORS: ectopia cordis, surgery.

\section{REFERÊNCIAS BIBLIOGRÁFICAS}

1 BALLINGER, W. F. - Pared toracica. In GIBBON Jr., J. H. Cirurgia toracica. Barcelona, Salvat Editores, 1971. cap. 13, p. $203-235$.

2 BARLOW, R. N. - Ectopia cordis cum sterni fissura. J. Pediatr. 2: 58-65, 1938.
A syndrome of congenital defects involving the abdominal wall, sternum, diaphragm pericardium, and heart. Surg. Gynecol. Obstet., 20: 602-614, 1958.

4 CRITTENDEN, I. H.; ADAMS, F. H.; MULDER, D. G. A syndrome featuring defects of the heart, sternum, diaphragm, and anterior abdominal wall. Circulation, 20: 396-404, 1959.

5 DOBELL, A. R. C.; WILLIAMS, H. B.; LONG, R. W. - 
ESCOBAR, M.; LIMA, R. C.; FRANÇA, N. A.; BELÉM, H.; SCHMIDT, E.; SOUZA, M. P.; ALECRIM, R.; DELLA SANTA, R.; MELLO, N.; GRANJA, L. G.; PONTES, J. - Ectopia "cordis": relato de 2 casos operados. Discussão do manuseio cirúrgico e revisão da literatura. Rev. Bras. Cir. Cardiovasc., 8 (3): 241-246, 1993.

Staged repair of ectopia cordis. J. Pediat. Surg., 17: 353-358, 1982.

6 ESCOBAR, M.; LIMA, R.; FRANÇA, N. A. C. - Ectopia cordis: a respeito de um caso. Rev. Cir. Cardiovasc., 1: 19-22, 1988.

7 GEVA, T.; VAN PRAAGH, S.; VAN PRAAGH, R. Thoracoabdominal Ectopia Cordis with Isolated Infundibular Atresia. Am. J. Cardiol., 66(10): 891$893,1990$.

8 GLASS, R. B. \& FERNBACH, S. K. - Ectopia cordis and the radiographic changes of new surgical repair technique. Clin. Radiol., 38: 443-444, 1987.

9

HARRISON, M.R.; FILLY, R. A.; STANGER, P.; LORIMIER, A. A. - Prenatal diagnosis and management of omphalocele and ectopia cordis. J. Pediatr. Surg., 17: 64-66, 1982.

10 JONES, A. F.; MCGRATH, R. L.; EDWARDS, S. M.; LILLY, J. R. - Immediate operation for ectopia cordis. Ann. Thorac. Surg., 28: 5, 484-6, 1979.

11 KAPLAN, C. L.; MATSUOKA, R.; GILBERT, E. F.; OPITZ, J. M.; KURNIT, D. M. - Ectopia cordis and cleft sternum: evidence for Mechanical Teratogenesis Following Rupture of the Chorion or Yolk Sac. Am. J. Med. Genet., vol. 21 (1), 187-202, 1985.

KHOURY, M. J.; CORDERO, J. F.; RASMUSSEN, S. Ectopia cordis and chromosome abnormalities: an epidemiologic perspective. Am. J. Med. Genet., 30: 811-817, 1988.

13 LECA, F.; THIBERT, M.; KHOURY, N.; FERMONT, L.; LABORDE, F.; DUMEZ, Y. - Extrathoracic heart (ectopia cordis): report of two cases and review of the literature. Int. J. Cardiol., 22 (2): 221, 1989.
14 LOGAN Jr., W. D.; CRISPIN, R. H.; PATTERSON, J. H.; ABBOT, O. A. - Ectopia cordis: report of case and discussion of surgical manegement. Pediat. Surg., 57: 898-900, 1965.

15 MILLHOUSE, R. F. \& JOOS, H. A. - Extrathoracic ectopia cordis: report of cases and review of literature. Am. Heart J., 57: 470-476, 1959.

16 MULDER, D. G.; CRITTENDEN, I. H.; ADAMS, F. H. Complete repair of a syndrome of congenital defects involving the abdominal wall, sternum, diaphragm, pericardium, and heart: excision of left ventricular diverticulum. Ann. Surg., 151: 113-122, 1960.

17 POULIAS, G. E.; RAFTOPOULOS, J.; POLEMIS, L.; SKOUTAS, B.; KATSOULI, R. - Total thoracic ectopia cordis with complete absence of sternum and pericardium and double diverticulum: case report. $J$. Cardiovasc. Surg., 23 (1): 75-78, 1982.

18 ROCCAFORTE, D. S.; MEHNERT, J. H.; PENICHE, A. - Repair of bifid sternum with autogenous cartilage: a case report. Ann. Surg., 149: 448-452, 1959.

19 SABISTON Jr., D. C. - The surgical management of bifid sternum with partial ectopia cordis. J. Thorac. Surg., 35: 118-122, 1958.

20 STARK, J. - Surgery for congenital heart defects. In STARK, J. \& DE LEVAL, M. (eds) New York, Grune \& Stratton cap. 39, pag. 1983. 506-507.

21 ULMER, H. E.; STOLZ, W.; KUHL, G.; MECHTERSHEIGER, G. - Ectopia cordis Report of a prenafally diagnosed cases and a short review of the literature. Monatsschr. Kinderheilkd. 137 (8): 468, 1989.

22 WATERSON, K. G.; WILKINSON, J. L.; KLIMAN, L.; MEE, R. B. - Thoracic ectopia cordis with doubleoutlet right ventricle: neonatal repair. Ann. Thorac. Surg., 53: 146-147, 1992. 